\title{
Quality Assessment of Moringa Seed Shells Based on Fingerprinting Using HPLC-DAD
}

\author{
Yanqin Zhu ${ }^{1,2}$, Ping $\mathrm{Du}^{2}$, Shaojun Huang ${ }^{2}$, Qinhong Yin $^{3}$ and Yaling Yang ${ }^{1 *}$ \\ Faculty of Life Science and Technology, Kunming University of Science and Technology, Kunming 650500, Yunnan, China \\ Research Center for Analysis and Measurement, Kunming University of Science and Technology, Kunming 650093, China \\ Yunnan Police College, Kunming 650223, China
}

Received: 05 September 2018; accepted: 12 December 2018

\begin{abstract}
A fingerprint analysis method was established for the quality control of Moringa seed shells by high-performance liquid chromatography with diode array detection (HPLC-DAD). The HPLC-DAD separation was performed on a Thermo Hypersil Gold C18 $(4.6 \mathrm{~mm} \times 250 \mathrm{~mm}, 5 \mu \mathrm{m})$ column by gradient elution with acetonitrile-water as mobile phase. The fingerprint of Moringa seed shells was established with good precision, reproducibility, and stability obtaining within $60 \mathrm{~min}$, and 13 common peaks in the fingerprint were designed. Similarity analysis, principal component analysis (PCA), and hierarchical clustering analysis (HCA) were carried out to analyze the obtained fingerprints. The similarity among 11 batches of samples in addition to No. 5 and 6 was no less than 0.92. Eleven samples could be classified into 2 clusters. The HPLC fingerprint technology and application of chemical pattern recognition can provide a more comprehensive reference for the quality control of medicinal plants.
\end{abstract}

Keywords: Moringa seed shells, fingerprint, similarity evaluation, PCA, HCA, HPLC-DAD

\section{Introduction}

Moringa oleifera belongs to the genus Moringaceae, which consists of 14 known species. Among these species, M. oleifera is the most widely known and utilized. The plant is a native of the sub-Himalayan regions of North-West India and is now indigenous to many countries in Africa, Arabia, South East Asia, the Pacific, the Caribbean islands, and South America $[1,2]$. The tree is prized mainly for its edible fruits, leaves, flowers, roots, and seed oil [3]. The root extract possesses antimicrobial activity [4]. The extracts from the flowers are found to have hepatoprotective effect [5]. Different parts of a M. oleifera tree contain 17 amino acids, protein, vitamin $\mathrm{C}$, glucosinolates, carotenoids, tocopherols, polyunsaturated fatty acids, and other nutritive compositions [6]. Moringa seed shells contain bioactive ingredients, such as polyphenols, flavonoids, and etc., and they are used in the prevention and treatment of diabetes, high blood pressure, skin disease, anemia, scurvy, obesity, arthritis, and other diseases [7-13]. The nutritive value and medicinal value of Moringa are getting more and more attention [14].

Medicinal plants have diversified types of components that vary in assay; therefore, quality control of these components remains a challenge. In recent years, chromatography methods, such as high-performance liquid chromatography (HPLC) [15-17], gas chromatography-mass spectrometry (GC-MS) [18], and HPLC-MS [19-21], have been used for quantitative analysis and quality control of Moringa. In 2013, Coppin and co-workers performed the detailed HPLC with ultraviolet and mass spectroscopy (HPLC-UV-MS) research that focused on establishing and comparing the flavonoid profiles in leaf samples from different African countries [19]. In addition, in 2016, Nouman et al. performed the assessment of polyphenolics in hydro-methanolic extracts obtained from the germplasm leaves of 7 cultivars of $M$. oleifera using HPLC

*Author for correspondence: ygs2013@yeah.net. with photodiode array detection and electrospray ionizationMS (HPLC-PDA-ESI-MS) analysis [20].

Fingerprinting techniques can provide an overall profile of almost every component of medicinal plants and are considered to be an important method for evaluating the quality of medicinal plants [22]. Many health organizations, including the U.S. Food and Drug Administration (FDA), the European Medicines Agency (EMEA), and the State Food and Drug Administration of China (SFDA), have recommended this strategy to assess the quality and consistency of botanical products. HPLC fingerprinting can be used to detect the complex constituents in plant extracts both qualitatively and quantitatively, and it is highly suitable for quality control of various plants. According to the literature published in the recent years, to the best of our knowledge, no literature study has been documented where a high-performance liquid chromatography fingerprint was used to evaluate the quality of Moringa seed shells.

This study aims to offer an efficient and affordable HPLC with diode array detection (HPLC-DAD) method for fingerprinting and reveal the correlation and consistency of quality control in Moringa seed shells preparations. The chemical fingerprints of Moringa seed shells from various regions were established and investigated by similarity analysis (SA), principal component analysis (PCA) and hierarchical clustering analysis (HCA). The combination of chromatographic fingerprint analysis afford a more comprehensive strategy for the quality evaluation of Moringa seed shells.

\section{Experimental}

2.1 Chemicals and Reagents. 4( $\alpha$-L-rhamnosyloxy)-benzyl isothiocyanate was purchased from Shanghai Ann Spectrum Experiment Technology Co., Ltd. (Shanghai, China). The purity of the component was determined to be higher than $98.0 \%$ by HPLC. The HPLC grade methanol, acetonitrile, phosphoric acid

This is an open-access article distributed under the terms of the Creative Commons Attribution-NonCommercial 4.0 International License (https://creativecommons.org/licenses/by-nc/4.0/), which permits unrestricted use, distribution, and reproduction in any medium for non-commercial purposes, provided the original author and source are credited, a link to the CC License is provided, and changes - if any - are indicated. 
Table 1. Representative samples of Moringa seed shells investigated in this study

\begin{tabular}{llc}
\hline Sample No. & Herbal species & Locations \\
\hline S1 & Moringa oleifera Lam. & Collection time \\
S2 & Moringa oleifera Lam. & Hot-Dry Valley, Sichuan Province \\
S3 & Moringa oleifera Lam. & 2015-9-11 \\
S4 & Moringa oleifera Lam. & Lum palmer bottom, Yunnan Province \\
S5 & Moringa oleifera Lam. & Baoshan Conggang, Yunnan Province \\
S6 & Moringa oleifera Lam. & Dehong palmer bottom, Yunnan Province \\
S7 & Moringa oleifera Lam. & Northwestward, India \\
S8 & Moringa oleifera Lam. & Kokang, Myanmar \\
S9 & Moringa oleifera Lam. & Dehong-11 \\
S10 & Moringa oleifera Lam. & 2015-9-20 \\
S11 & Moringa oleifera Lam. & Lucheng Jiang Ba, Yunnan Province \\
& & Baoshan, Yunnan Province \\
\end{tabular}

and acetic acid were purchased from Merck (Merck Co., Darmstadt, Germany). Deionized water was purified by a MilliQ system from Millipore (Bedford, MA, USA). Other chemicals and solvents used in sample preparation were of analytical grade.

2.2 Plant Materials. Moringa seed samples were collected respectively from different areas (Table 1). Then, the samples were processed, decorticated, and pulverized for later use.

2.3 Instrumentation and Chromatographic Conditions. HPLC fingerprint analysis was performed on a DIONEX U3000 system (DIONEX Corp., USA), equipped with a dual ternary solvent manager, an auto-sampler, and a diode array detector, and a class-up station was used for liquid chromatographic analysis. Chromatographic data were accessed, processed, and handled, employing Chromeleon 7 (DIONEX Corp., USA) software. The influence of some chromatographic conditions for the HPLC analysis, such as column length, column temperature, wave length and mobile phase was investigated. Different types of columns were tested. Thermo Hypersil Gold C18 column is better than that of Agilent Eclipse XDB-C18 column. A SB3200 ultrasonic cleaning instrument (Branson Ultrasonic Instrument Co., Shanghai, China) was used for extraction. Comparing the chromatograms at 3 different temperatures, namely, $25{ }^{\circ} \mathrm{C}, 30{ }^{\circ} \mathrm{C}$, and room temperature, we found no obvious separation difference, but after overall consideration of the analysis time and separating effect, the column temperature was set at room temperature.

Selection of an appropriate detection wavelength was of great importance to ensure precise detection of some essential constituents and to achieve more peaks. DIONEX U3000 with DAD was used in the analysis, and full scan runs were made first to choose the optimum wavelength that provided the best results in chromatographic fingerprinting analysis. Chromatogram at $203 \mathrm{~nm}$ showed the most abundant component information and the steadiest baseline, compared with the other wavelengths. Ultimately, we selected $203 \mathrm{~nm}$ as the monitoring wavelength.

The effect of the composition of the mobile phase in the chromatographic separation of the samples was researched in this study. Different mobile phases were tried, such as acetonitrile-phosphoric acid/water $(1: 100, v / v)$, methanol-acetic acid/ water $(1: 100, v / v)$, methanol-water, acetonitrile-water, etc. Finally, acetonitrile-water was selected as an appropriate mobile phase with gradient elution. The gradient program was set as follows: $0-20 \mathrm{~min}, 10-30 \%$ (A); $20-40 \mathrm{~min}, 30-50 \%$ (A); 40-45 min, 50-90\% (A); 45-50 min, 90\% (A); 50-52 min, 90-10\% (A); $52-60 \mathrm{~min}, 10 \%$ (A). The injection volume of each sample, as well as standard solution, was $5 \mu \mathrm{L}$, and the flow rate was $1.0 \mathrm{~mL} / \mathrm{min}$.

2.4 Sample Preparation. All samples were ground to fine powder (40 mesh) using a pulverizer. The 11 pulverized samples of Moringa seed shells from different locations were accurately weighed (approximately $5.0 \mathrm{~g}$ ), soaked for $24 \mathrm{~h}$ in $30 \mathrm{~mL}$ of $50 \%$ methanol, and extracted by an ultrasonic cleaner at room temperature with $30 \mathrm{~min}$. The extracted solutions were filtered through a $0.45-\mu \mathrm{m}$ filter membrane prior to injection into the HPLC-DAD system.

2.5 Similarity Analysis. Characteristic peaks were present in all chromatograms of detected samples. Identification of the characteristic peaks and similarities of the fingerprint data were performed on the basis of the relative retention time (RRT) and relative peak area (RPA) using the professional software named Similarity Evaluation System for Chromatographic Fingerprint of Traditional Chinese Medicine (2004A). The software was used to employ the correlative coefficient in evaluating the similarities of different chromatogram. Matching among the fingerprints of samples was performed by a multipoint calibration mode based on the retention time and UV spectra. In this test, all 11 samples were examined to generate a reference chromatogram as the representative standard fingerprint, and the similarity of each chromatogram against this standard chromatogram was then calculated. Furthermore, the RRT and RPA of each characteristic peak related to a reference peak were also constructed for quantitative measurement of the chemical composition of Moringa seed shells samples.

2.6 Hierarchical Cluster Analysis. Hierarchical clustering analysis is a multivariate analytical technique used to classify samples into different groups. In this study, the Moringa seed shells samples were grouped by using Statistics software with the v.18.0 SPSS system (SPSS Inc., USA) to discover the differences in samples caused by space factors.

\section{Results and Discussion}

3.1 Optimization of Extracting Conditions. To achieve satisfactory extraction efficiency, different extraction conditions were studied by comparing reflux extraction, Soxhlet extraction, ultrasonic extraction, and soak extraction methods. According to the temperature effect and the withdrawal rates of $4(\alpha$-L-rhamnosyloxy $)$ benzyl isothiocyanate, the soak extraction of $24 \mathrm{~h}$ and the ultrasonic extraction for $30 \mathrm{~min}$ were chosen as the extracting method. Meanwhile, various extraction solutions such as petroleum ether (boiling range: $30-60{ }^{\circ} \mathrm{C}$ ), $n$-hexane, acetone, and $50 \%$ methanol were studied. As a result, the optimal extraction conditions are as follows: soak extraction of $24 \mathrm{~h}$ and then ultrasonic extraction for $30 \mathrm{~min}$ in $50 \%$ methanol.

3.2 Methodology Validation. All tests below were carried out on the sample extract solutions prepared as described in the section Sample Preparation. The method precision was evaluated by carrying out 6 independent HPLC runs of the same sample solution and by analyzing fingerprints of 5 parallel extraction solutions of the same sample. Precision of sample stability was determined with measurements from a single sample solution stored at room temperature for $0,2,4$, 6,8 , and $12 \mathrm{~h}$. The reproducibility of the experiment was evaluated by analyzing 6 separate extract solutions of one sample. RRT and RPA of each characteristic peak were calculated for the estimation of precision, stability, and 


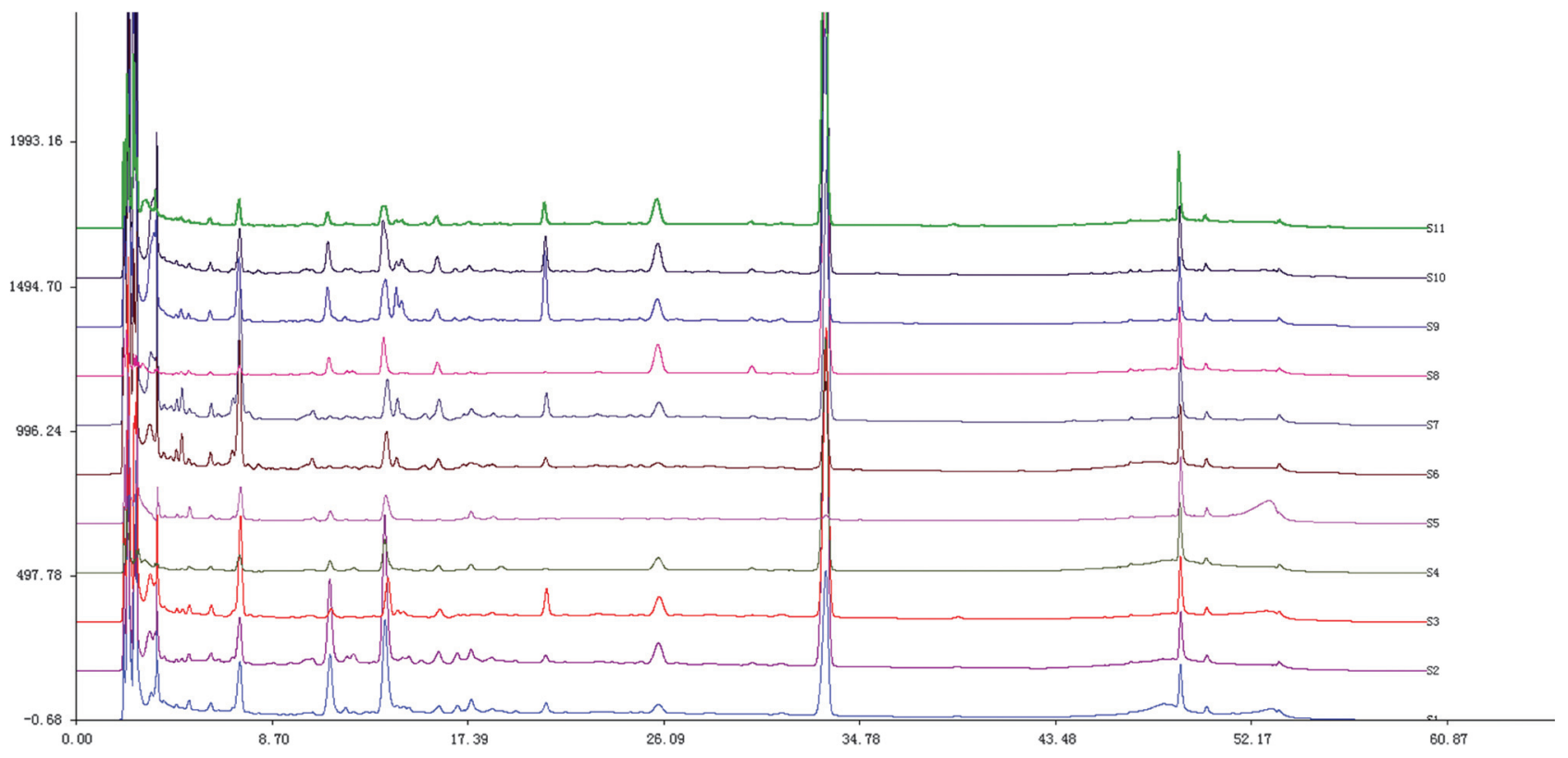

Figure 1. Chromatogram of the fingerprints of the 11 samples

reproducibility, and all the results of the relative standard deviation (RSD) of RRT and RPA were found not to exceed $3.0 \%$. Therefore, all results demonstrated that the quality of the studied samples and the HPLC-DAD measurements were stable and under control.

3.3 Analysis of High-Performance Liquid Chromatography Fingerprint. The 11 sample solutions of Moringa seed shells and standard substances were prepared and analyzed, and chromatograms of the samples were recorded in $60 \mathrm{~min}$, which is shown in Figure 1. According to the results of HPLC fingerprint, we found 13 common characteristic peaks. Among them, peak 11 at retention time of $33.28 \mathrm{~min}$, which was designated as the reference peak. Because it was present with a stable time and a large peak area in the chromatogram, the RSD value is below $3.0 \%$. Furthermore, its comparative standing time of common peaks approximately fits the requirements of "Technological Requirements of Fingerprint Chromatography". Consequently, the established fingerprint method for Moringa seed shells is convenient and feasible as a tool for species authentication and quality assessment of the raw material.

3.4 Relative Peak Area and Similarity of the Sample. The 11 sample solutions of Moringa seed shells were prepared and analyzed, and chromatograms of the samples were recorded at $203 \mathrm{~nm}$. The chromatographic peaks in different samples with the same relative retention time were defined as the characteristic peaks. Thirteen peaks were identified as the characteristic peaks. Among the characteristic peaks, peak 11 at retention time of 33.28 min was designated as the reference peak for the calculation of RPA. On the basis of this reference, RRT and RPA of these 13 peaks in 11 samples were shown in Table 2. The data of the chromatographic fingerprints were imported into the Similarity Evaluation System for Chromatographic Fingerprint of Traditional Chinese medicine (TCM) (Version 2004 A) software. In this study, a similarity analysis based on matching against the median of the fusion vectors of all the samples was performed. The reference chromatogram was generated by the median method. As a result, the similarity indexes of the 11 samples are listed in Table 3.

3.5 Identification of Chromatographic Peaks. Standard substances coupled with HPLC-DAD had been generally used for qualitative or quantitative analysis of components in analytes separated. In this study, 4( $\alpha$-L-rhamnosyloxy) benzyl isothiocyanate (peak 11) was explicitly identified in the samples of Moringa seed shells. The other compounds of common peaks were needed to be identified in a further study. The HPLC chromatogram of sample No.1 obtained at $203 \mathrm{~nm}$ was shown in Figure 2.

Compared with the reference chromatogram generated by the median method, the similarity indexes of the 11 samples in addition to No. 5 and 6 were all above 0.92 (Table 3). It showed that 9 batch Moringa seed shells samples had a high similarity matching, which means that the qualities of 9 batch Moringa seed shells samples collected from different locations

Table 2. The calculation result of the relative peak area of the communion peak

\begin{tabular}{|c|c|c|c|c|c|c|c|c|c|c|c|c|c|}
\hline \multirow[t]{2}{*}{ RRT } & \multirow[t]{2}{*}{ RSD (\%) } & \multicolumn{11}{|c|}{ RPA } & \multirow[t]{2}{*}{ RSD (\%) } \\
\hline & & $\overline{\mathrm{S} 1}$ & $\mathrm{~S} 2$ & S3 & S4 & S5 & S6 & S7 & $\mathrm{S} 8$ & S9 & S10 & $\overline{S 11}$ & \\
\hline 0.108 & 0.66 & 0.119 & 0.047 & 0.047 & 0.003 & 2.466 & 0.324 & 0.124 & 0.001 & 0.046 & 0.031 & 0.013 & 71.60 \\
\hline 0.150 & 2.22 & 0.028 & 0.010 & 0.014 & 0.012 & 1.593 & 0.029 & 0.009 & 0.004 & 0.014 & 0.005 & 0.002 & 44.86 \\
\hline 0.180 & 0.36 & 0.032 & 0.012 & 0.020 & 0.009 & 0.595 & 0.087 & 0.028 & 0.003 & 0.019 & 0.010 & 0.010 & 44.57 \\
\hline 0.218 & 0.30 & 0.227 & 0.082 & 0.211 & 0.047 & 6.059 & 0.931 & 0.319 & 0.011 & 0.159 & 0.070 & 0.044 & 68.47 \\
\hline 0.338 & 0.45 & 0.342 & 0.203 & 0.027 & 0.035 & 2.448 & 0.018 & 0.007 & 0.030 & 0.086 & 0.065 & 0.028 & 112.58 \\
\hline 0.412 & 0.53 & 0.548 & 0.355 & 0.108 & 0.122 & 7.926 & 0.381 & 0.142 & 0.065 & 0.157 & 0.138 & 0.061 & 71.00 \\
\hline 0.483 & 0.29 & 0.052 & 0.035 & 0.025 & 0.022 & 0.288 & 0.126 & 0.069 & 0.022 & 0.042 & 0.037 & 0.023 & 44.22 \\
\hline 0.527 & 0.66 & 0.064 & 0.027 & 0.001 & 0.020 & 1.418 & 0.022 & 0.018 & 0.002 & 0.007 & 0.008 & 0.007 & 83.87 \\
\hline 0.627 & 0.38 & 0.056 & 0.019 & 0.068 & 0.007 & 0.173 & 0.093 & 0.074 & 0.001 & 0.175 & 0.061 & 0.040 & 99.94 \\
\hline 0.776 & 0.22 & 0.090 & 0.086 & 0.092 & 0.077 & 0.333 & 0.085 & 0.094 & 0.092 & 0.115 & 0.105 & 0.097 & 53.69 \\
\hline 1.000 & 0.07 & 1.000 & 1.000 & 1.000 & 1.000 & 1.000 & 1.000 & 1.000 & 1.000 & 1.000 & 1.000 & 1.000 & 52.17 \\
\hline 1.473 & 0.05 & 0.152 & 0.076 & 0.103 & 0.134 & 9.178 & 0.383 & 0.135 & 0.072 & 0.119 & 0.084 & 0.093 & 8.19 \\
\hline 1.508 & 0.05 & 0.030 & 0.013 & 0.016 & 0.017 & 1.634 & 0.062 & 0.020 & 0.009 & 0.017 & 0.010 & 0.010 & 12.62 \\
\hline
\end{tabular}


Table 3. Similarity comparison of chromatographic patterns of the 11 samples

\begin{tabular}{llllllllcccccc}
\hline No. & S1 & S2 & S3 & S4 & S5 & S6 & S7 & S8 & S9 & S10 & S11 & Reference \\
\hline S1 & 1.000 & 0.973 & 0.896 & 0.888 & 0.538 & 0.825 & 0.900 & 0.857 & 0.917 & 0.904 & 0.864 & 0.929 \\
S2 & 0.973 & 1.000 & 0.951 & 0.962 & 0.369 & 0.769 & 0.935 & 0.947 & 0.963 & 0.971 & 0.948 & 0.978 \\
S3 & 0.896 & 0.951 & 1.000 & 0.984 & 0.294 & 0.830 & 0.991 & 0.977 & 0.991 & 0.989 & 0.985 & 0.994 \\
S4 & 0.888 & 0.962 & 0.984 & 1.000 & 0.252 & 0.744 & 0.958 & 0.996 & 0.978 & 0.996 & 0.996 & 0.991 \\
S5 & 0.538 & 0.369 & 0.294 & 0.252 & 1.000 & 0.659 & 0.372 & 0.165 & 0.316 & 0.247 & 0.192 & 0.324 \\
S6 & 0.825 & 0.769 & 0.830 & 0.744 & 0.659 & 1.000 & 0.892 & 0.701 & 0.811 & 0.758 & 0.728 & 0.810 \\
S7 & 0.900 & 0.935 & 0.991 & 0.958 & 0.372 & 0.892 & 1.000 & 0.944 & 0.979 & 0.966 & 0.957 & 0.981 \\
S8 & 0.857 & 0.947 & 0.977 & 0.996 & 0.165 & 0.701 & 0.944 & 1.000 & 0.969 & 0.993 & 0.998 & 0.981 \\
S9 & 0.917 & 0.963 & 0.991 & 0.978 & 0.316 & 0.811 & 0.979 & 0.969 & 1.000 & 0.990 & 0.979 & 0.993 \\
S10 & 0.904 & 0.971 & 0.989 & 0.996 & 0.247 & 0.758 & 0.966 & 0.993 & 0.990 & 1.000 & 0.996 & 0.996 \\
S11 & 0.864 & 0.948 & 0.985 & 0.996 & 0.192 & 0.728 & 0.957 & 0.998 & 0.979 & 0.996 & 1.000 & 0.986 \\
Reference & 0.929 & 0.978 & 0.994 & 0.991 & 0.324 & 0.810 & 0.981 & 0.981 & 0.993 & 0.996 & 0.986 & 1.000 \\
\hline
\end{tabular}

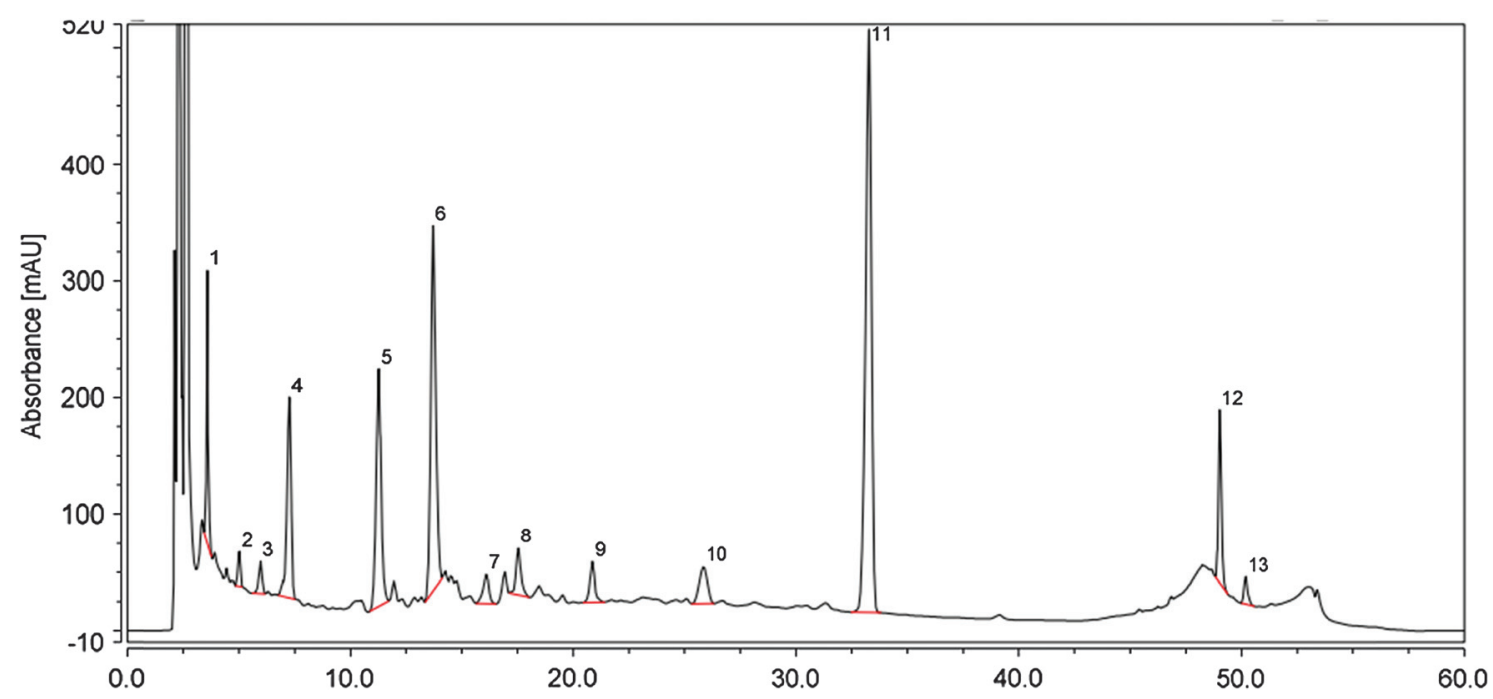

Figure 2. The HPLC chromatogram of sample No. 1 acquired at $203 \mathrm{~nm}$

were stable and consistent. The 13 characteristic peaks in chromatograms were the main components of Moringa seed shells. In practice of quality control, the herbs which have the smallest similarity indexes or are below a certain value can be regarded as not qualified. Consequently, for quality control of Moringa seed shells, 0.90 was set as a suitable value, and it is easy to find that the samples of Moringa seed shells in addition to No. 5 and 6 with similarity indexes above 0.92 have credible qualities.

Table 4. Total variance explained

\begin{tabular}{|c|c|c|c|}
\hline \multirow[t]{2}{*}{ Components } & \multicolumn{3}{|c|}{ Initial eigenvalues } \\
\hline & Total & $\%$ of variance & Cumulative $\%$ \\
\hline$\overline{\mathrm{p} 1}$ & 4.699 & 36.149 & 36.149 \\
\hline p 2 & 3.266 & 25.127 & 61.276 \\
\hline p 3 & 3.208 & 24.678 & 85.953 \\
\hline p 4 & 0.957 & 7.364 & 93.317 \\
\hline p 5 & 0.388 & 2.985 & 96.302 \\
\hline p 6 & 0.213 & 1.641 & 97.943 \\
\hline p 7 & 0.144 & 1.110 & 99.054 \\
\hline p 8 & 0.082 & 0.630 & 99.684 \\
\hline p 9 & 0.034 & 0.262 & 99.946 \\
\hline p 10 & 0.007 & 0.054 & 100.000 \\
\hline p 11 & $8.245 \mathrm{E}-17$ & $6.342 \mathrm{E}-16$ & 100.000 \\
\hline p 12 & $5.173 \mathrm{E}-17$ & $3.979 \mathrm{E}-16$ & 100.000 \\
\hline p 13 & $-2.877 \mathrm{E}-17$ & $-2.213 \mathrm{E}-16$ & 100.000 \\
\hline \multirow{2}{*}{ Components } & \multicolumn{3}{|c|}{ Extraction sums of squared loadings } \\
\hline & Total & $\%$ of variance & Cumulative $\%$ \\
\hline 1 & 4.699 & 36.149 & 36.149 \\
\hline 2 & 3.266 & 25.127 & 61.276 \\
\hline 3 & 3.208 & 24.678 & 85.953 \\
\hline
\end{tabular}

3.6 PCA. PCA is an effective data technique, which has long been used as an exploratory method to study the classification of samples [23-28]. PCA was employed to analyze the relationship of the 11 Moringa seed shells samples from different sources, implemented by performing singular value decomposition on the data array of areas of the 13 common peaks (Table 2 and 3), each row representing a sample and each column containing the values of 13 peak areas. As is shown in Tables 4 and 5, 3 components were extracted. It is apparent that the 3 extracted components explain $100 \%$ of the variance of the data matrix and represent the dominant information. With these figures, differences between the 3 groups were discovered according to principal

Table 5. Component matrix

\begin{tabular}{lccc}
\hline & \multicolumn{3}{c}{ Components } \\
\cline { 2 - 4 } & 1 & 2 & 3 \\
\hline Zscore(p1) & 0.691 & 0.647 & 0.199 \\
Zscore(p2) & 0.721 & -0.301 & -0.257 \\
Zscore(p3) & 0.522 & 0.780 & 0.228 \\
Zscore(p4) & 0.504 & 0.843 & -0.025 \\
Zscore(p5) & 0.461 & -0.490 & 0.720 \\
Zscore(p6) & 0.557 & -0.358 & 0.725 \\
Zscore(p7) & -0.048 & 0.556 & 0.763 \\
Zscore(p8) & .659 & -0.526 & 0.385 \\
Zscore(p9) & -0.088 & 0.563 & 0.306 \\
Zscore(p10) & -0.660 & 0.041 & 0.701 \\
Zscore(p11) & -0.642 & -0.043 & 0.709 \\
Zscore(p12) & -0.793 & 0.390 & -0.277 \\
Zscore(p13) & 0.855 & 0.110 & -0.333 \\
Extraction method: principal component analysis. Three components \\
extracted.
\end{tabular}




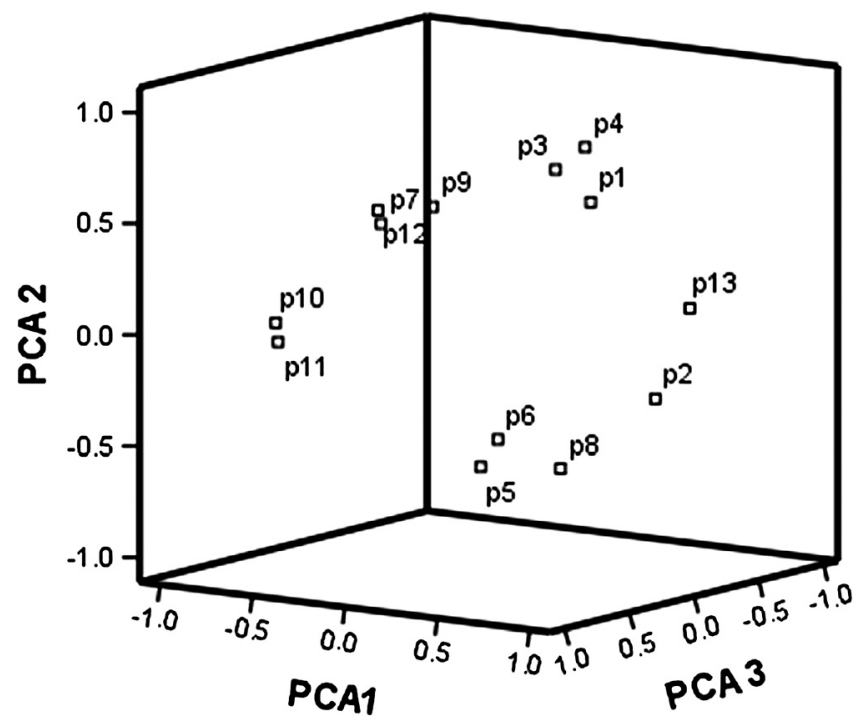

Figure 3. PCA score figure

component analysis (Figure 3). It indicates that PCA combined with similarity analysis can be used for discrimination and classification of Moringa seed shell samples.

3.7 HCA. The areas of 13 characteristic peaks were selected for HCA. As we know, hierarchical clustering can efficiently organize biological measurements and produce a qualitative and quantitative representation of original experimental results through statistical organization and graphic display [29-33]. In the analysis, the peak areas of the 13 characteristic peaks of samples 1-11 formed a matrix of 11 $\times 13$. Similarities among samples $1-11$ were calculated using the Statistics software. It was clear that the samples could be divided into 2 clusters at a rescaled distance of 25: samples 5 and 6 in cluster 1, and the others could be grouped into cluster 2. HCA provided a quantitative comparison of the samples. The dendrogram constructed according to hierarchical cluster analysis, based on the squared Euclidean distance, shows the relationships among the Moringa seed shells samples collected from 11 locations (Figure 4).

The findings of HCA show that there was a larger difference of chemical components between India and Myanmar because of the size of Moringa seeds. Similar to the appearance

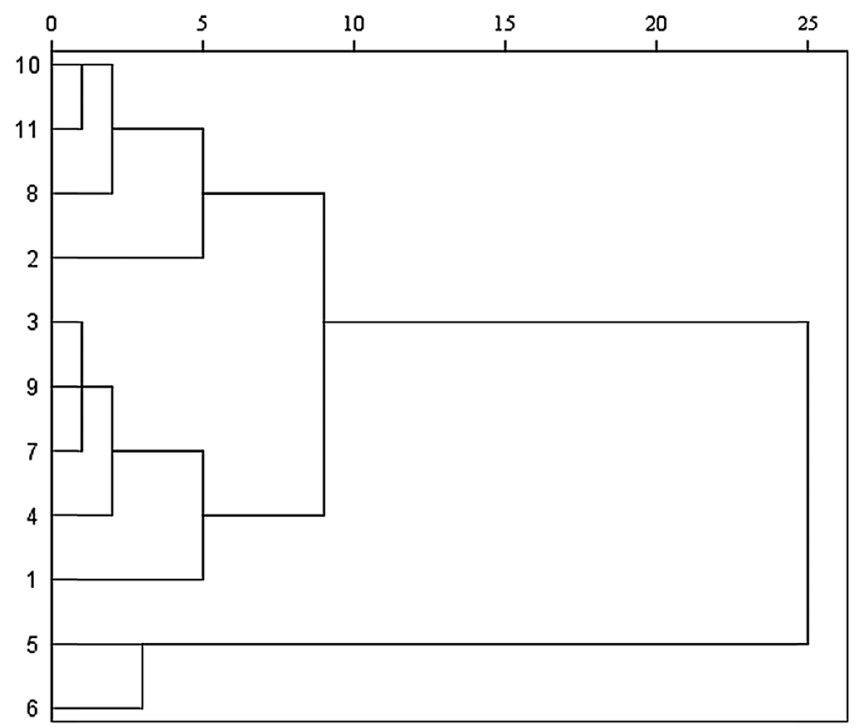

Figure 4. Results of hierarchical cluster analysis of 11 samples analysis, the hierarchical cluster analysis in this study also demonstrates the high similarity in the different areas. Hence, the researching results of hierarchical cluster analysis and similarity analysis are consistent. Meanwhile, hierarchical cluster analysis proved that the 11 samples could be separated into 2 distinct groups based on their compositional fingerprints. This finding further suggests that HCA represents an effective method for the categorization of the Moringa seed shells.

\section{Conclusions}

In conclusion, the chemical fingerprints of 11 batches of Moringa seed shells samples from various sources were established using HPLC-DAD. Eleven samples were performed by this method, and the chromatographic data were processed by similarity analysis and hierarchical cluster analysis. The result of the similarity analysis shows that Moringa seed shells samples have relatively constant quality, and this HPLC-DAD fingerprinting method could be applied in the quality control as a powerful tool. The result of hierarchical cluster analysis indicates that the components in Moringa seed shells from the 11 districts have very similar contents. The HPLC-DAD fingerprinting method of Moringa seed shells established in this paper is precise, efficient, practical, and reliable. As a result, the HPLC fingerprint technology could be applied further to evaluate and control effectively the quality of Moringa seed shells. Similarly, the strategy of qualitative and quantitative reliability analysis is the most promising for the assessment of the investigated plant material.

\section{References}

1. Abdulkarim, S. M.; Long, K.; Lai, O. M.; Muhammad, S. K. S.; Ghazali, H. M. Food Chem. 2005, 93, 253-263.

2. Palafox, J. O.; Navarrete, A.; Sacramento-Rivero, J. C.; Rubin-Atoche, C.; Escoffie, P. A.; Rocha-Uribe, J. A. Am. J. Anal. Chem. 2012, 3, 946-949.

3. Sanchez-Machado, D. I.; Nunez-Gastelum, J. A.; Reyes-Moreno, C.; Ramirez-Wong, B.; Lopez-Cervantes, J. Food Anal. Method 2010, 3, 175-180. 4. Abdelgadir, H. A.; Plooy, C. P.; Ndhlala, A. R. S. Afr. J. Bot. 2017, 109, 324-324

5. Fernandes, E. E.; Pulwale, A. V.; Patil, G. A.; Moghe, A. S. Pharmacognosy Res. 2016, 8, 231-237.

6. Saini, R. K.; Sivanesan, I.; Keum, Y. S. Biotech. 2016, 6, 203.

7. Al-Malki, A. L.; Rabey, H. A. E. Biomed. Res. Int. 2015, 2015, 1-13.

8. Aviara, N. A.; Musa, W. B.; Owolarafe, O. K.; Ogunsina, B. S.; Oluwole, F. A. J. Food Sci. Technol. 2015, 52, 4499-4506.

9. Ijarotimi, O. S.; Adeoti, O. A.; Ariyo, O. Food Sci. Nutr. 2013, 1, 452-463. 10. Jung, I. L. Plos One 2014, 9, 1-10.

11. Mahajan, S. G.; Meht, A. A. J. Herbs Spices Med. Plants 2009, 15, 149-163.

12. Mbikay, M. Front. Pharmacol. 2012, 3, 1-12.

13. Tiloke, C.; Phulukdaree, A.; Chuturgoon, A. A. Altern. Med. 2013, 13 , 226-233.

14. Stohs, S. J.; Hartman, M. J. Phytother. Res. 2015, 29, 796-804.

15.Shanker, K.; Gupta, M. M.; Srivastava, S. K.; Bawankule, D. U.; Pal, A.; Khanuja, S. P. S. Food Chem. 2007, 105, 376-382.

16. Vongsak, B.; Sithisarn, P.; Mangmool, S.; Thongpraditchote, S.; Wongkrajang, Y.; Gritsanapan, W. Ind. Crop. Prod. 2013, 44, 566-571.

17. Pachauri, S. D.; Khandelwal, K.; Singh, S. P.; Sashidhara, K. V.; Dwivedi, A. K. Med. Chem. Res. 2013, 22, 5284-5289.

18. Khan, W.; Parveen, R.; Chester, K.; Parveen, S.; Ahmad, S. Front. Pharmacol. 2017, 8, 577 .

19. Coppin, J. P.; Xu, Y.; Chen, H.; Pan, M. H.; Ho, C. T.; Juliani, R.; Simon, J. E.; Wu, Q. J. Funct. Foods 2013, 5, 1892-1899.

20. Nouman, W.; Anwar, F.; Gull, T.; Newton, A.; Rosa, E.; DomínguezPerles, R. Ind. Crop. Prod. 2016, 83, 166-176.

21. Rodríguez-Pérez, C.; Gilbert-López, B.; Mendiola, J. A.; QuirantesPiné, R.; Segura-Carretero, A.; Ibañez, E. Electrophoresis 2016, 37, 1938-1946. 22.Alaerts, G.; Pieters, S.; Logie, H.; Erps, J. V.; Merino-Arévalo, M.; Dejaegher, B.; Smeyers-Verbeke, J.; Heyden, Y. V. J. Pharm. Biomed. Anal. 2014, 95, 34-46.

23. Wiberg, K.; Andersson, M.; Hagman, A.; Jacobsson, S. P. J. Chromatogr. A 2004, 1029, 13-20.

24. Wiberg, K. J. Chromatogr. A 2006, 1108, 50-67.

25. Zhou, Y.; Xu, G.; Choi, F. F. K.; Ding, L. S.; Han, Q. B.; Song, J. Z.; Qiao, C. F.; Zhao, Q. S.; Xu, H. X., J. Chromatogr. A 2009, 1216, 4847-4858.

26. Gao, W.; Gray, N.; Heaton, J.; Smith, N. W.; Jia, Y.; L-Quigley, C. J. Chromatogr. A 2012, 1228, 324-328.

27. Gao, P. P.; Shi, B.; Li, Z. C.; Wang, P.; Yin, C. C.; Yin, Y. J.; Zan, L. S. Food Anal. Method 2018, 11, 23-33. 
28. Esteki, M.; Farajmand, B.; Kolahderazi, Y.; Simal-Gandara, J. Food Anal. Method 2017, 10, 3312-3320.

29. Peng, L.; Wang, Y. Z.; Zhu, H. B.; Chen, Q. M. Food Chem, 2011 $25,1064-1071$

30. Lima, D. C.; Santos, A. M. P.; Araujo, R. G. O.; Scarminio, I. S.; Bruns, R. E.; Ferreira, S. L. C. Microchem. J. 2010, 95, 222-226.
31. Patras, A.; Brunton, N. P.; Downey, G.; Rawson, A.; Warriner, K.; Gernigon, G. J. Food Compos. Anal. 2011, 24, 250-256.

32. Perez-Martin, L.; Bustamante-Rangel, M.; Delgado-Zamarreno, M. M. Food Anal. Method 2017, 10, 1191-1201.

33. Yu, X. X.; Liu, Q. D.; Xie, Z. S.; Lam, S. C.; Xu, X. J. Food Anal. Method 2015, 8, 1868-1875. 